\title{
Prognostic value of the density of tumor-infiltrating lymphocytes in colorectal cancer liver metastases
}

\author{
EN WANG ${ }^{1}$, MASATSUNE SHIBUTANI ${ }^{1}$, HISASHI NAGAHARA ${ }^{1}$, TATSUNARI FUKUOKA ${ }^{1}$, \\ YASUHITO ISEKI $^{1}$, YUKI OKAZAKI ${ }^{1}$, SHINICHIRO KASHIWAGI ${ }^{2}$, \\ HIROAKI TANAKA $^{1}$ and KIYOSHI MAEDA ${ }^{3}$ \\ Departments of ${ }^{1}$ Gastroenterological Surgery and ${ }^{2}$ Breast and Endocrine Surgery, Osaka City University \\ Graduate School of Medicine, Abeno-ku, Osaka 545-8585; ${ }^{3}$ Department of Gastroenterological \\ Surgery, Osaka City General Hospital, Miyakojima-ku, Osaka 534-0021, Japan
}

Received June 12, 2021; Accepted September 24, 2021

DOI: $10.3892 / \mathrm{ol} .2021 .13098$

\begin{abstract}
Tumor-infiltrating lymphocytes (TILs) have been reported to reflect the anti-tumor immune status of patients and to be correlated with their prognosis and therapeutic outcomes. However, the characteristics of the local immune status in metastatic tumors is poorly understood, as primary tumors have been the focus in most previous studies. In addition, the local immune status may be influenced by preoperative chemotherapy. The present study aimed therefore to investigate the relationship between the degree of TIL infiltration and the prognosis in patients with curative resection of colorectal cancer liver metastases and to examine the effects of preoperative chemotherapy on the function of immune cells. A total of 108 patients who underwent curative resection of colorectal cancer liver metastases in our department between May 1996 and January 2017 were enrolled in the present study. Peripheral blood samples were obtained within two weeks before surgery. TIL infiltration was evaluated by immunohistochemical staining of surgically resected specimens of liver metastases using anti-CD8/CD3 antibodies. The mean number of TILs in five different fields was calculated, and patients were classified into a high-TIL group and a low-TIL group. Furthermore, patients were divided into three groups as follows: i) A group of patients who did not receive preoperative chemotherapy; ii) a group of patients who received short-term preoperative chemotherapy for $<6$ months; and iii) a group of
\end{abstract}

Correspondence to: Dr Masatsune Shibutani, Department of Gastroenterological Surgery, Osaka City University Graduate School of Medicine, 1-4-3 Asahi-machi, Abeno-ku, Osaka 545-8585, Japan E-mail: fbxbj429@ybb.ne.jp

Abbreviations: HPF, high-power field; MST, median survival time; OS, overall survival; RFS, relapse-free survival; ROC, receiver operating characteristic; TILs, tumor-infiltrating lymphocytes

Key words: CD8, CD3, tumor-infiltrating lymphocytes, peripheral lymphocyte, colorectal cancer, liver metastasis, prognosis patients who received long-term preoperative chemotherapy for $\geq 6$ months. The results demonstrated that the density of TILs in colorectal liver metastases was not correlated with the absolute peripheral lymphocyte count in all patients. Furthermore, the degree of CD8 ${ }^{+}$TIL infiltration in liver metastases was significantly lower in the recurrence group compared with the recurrence-free group following hepatectomy. In all patients with colorectal liver metastases, the degree of CD8 ${ }^{+} \mathrm{TIL}$ infiltration was significantly associated with the relapse-free and overall survival. In patients without preoperative chemotherapy, the degree of CD8 ${ }^{+}$TIL infiltration was significantly associated with the relapse-free survival, and a high CD8 ${ }^{+} \mathrm{TIL}$ presence tended to have a better effect on the overall survival than a low CD8 ${ }^{+}$TIL presence. In the short-term chemotherapy group, the degree of CD8 ${ }^{+} \mathrm{TIL}$ infiltration was significantly associated with the relapse-free and overall survival. In the long-term chemotherapy group, there were no significant differences between the high- and low- CD8 ${ }^{+}$TIL groups in the relapse-free and overall survival. In contrast to CD8 ${ }^{+}$TILs, CD3 ${ }^{+}$TILs showed a poor prognostic ability. In summary, the degree of CD8 $8^{+} \mathrm{TIL}$ infiltration in colorectal cancer liver metastases may be correlated with patient prognosis. However, in patients who received long-term chemotherapy before surgery, the degree of TIL infiltration was not necessarily associated with prognosis as the anti-tumor effects of TILs may decrease. The degree of CD8 ${ }^{+}$TIL infiltration may therefore be considered as a useful prognostic factor in patients with colorectal liver metastases, but the prognostic accuracy may decrease in patients who received long-term chemotherapy.

\section{Introduction}

Colorectal cancer was the third-most common cancer worldwide in 2018 after lung and breast cancers, and was characterized by the second highest mortality rate after lung cancer (1). The liver is the most common site of synchronous distant metastasis of colorectal cancer, which appears in $10.9 \%$ of patients (2), and the liver is the most common site of recurrence of colorectal cancer, which is observed in $7.1 \%$ of patients (2).

The most common metastatic organ is the liver, as most of the intestinal mesenteric drainage spreads to the liver via 
the portal vein. Hepatectomy may therefore help achieving curative treatment of colorectal liver metastasis (2-5). Curative resection, which is recommended for liver metastases when the primary tumor and extrahepatic metastases have been controlled or can be controlled, has been reported to result in a better prognosis than other treatments $(2,6,7)$.

Previous studies have demonstrated that the tumor microenvironment plays an important role in cancer progression. Tumor-infiltrating lymphocytes (TILs), which represent one type of immune cells in the tumor microenvironment, can reflect the anti-tumor immune status of the patients and are positively correlated with the prognosis of various types of cancer, including colorectal cancer (8-12). Furthermore, it has been reported that the degree of TIL infiltration is positively correlated with the therapeutic outcomes after chemotherapy and radiotherapy and might be considered as a useful predictive marker for treatment success (13-16). TILs may therefore serve a crucial role in cancer progression.

Because primary tumors have been the focus of most previous studies, little is known about the characteristics of the local immune status in metastatic tumors. However, the characteristics of local immunity in metastatic tumor have been gradually elucidated in the recent years. Lee et al (17) reported a positively correlation between the degree of TIL infiltration in primary tumor of colorectal cancer and that in liver and lung metastases. In another study, the activation state of local immunity, including activation marker and suppression marker, in colorectal cancer liver metastases was demonstrated to be similar to that of the primary lesion resected at the same time (18). Furthermore, it has been shown that TILs in liver metastases are positively correlated with tumor doubling time (19). However, the relationship between the local immune status in colorectal cancer liver metastases and patient prognosis remains unclear, although the characteristics of local immunity in colorectal cancer liver metastases have gradually been elucidated. In addition, chemotherapy, which is often given before resection of liver metastases, has been reported to potentially alter the local immune status (20).

The present study aimed to investigate the relationship between the degree of TIL infiltration and the prognosis in patients who underwent curative resection of colorectal cancer liver metastases and to examine the effects of preoperative chemotherapy on the function of immune cells.

\section{Materials and methods}

Patients. A total of 108 patients with colorectal cancer who underwent curative resection of colorectal cancer liver metastases at the department of Surgery, Osaka City University (Osaka, Japan) between May 1996 and January 2017 were enrolled in the present study. Cases with hepatic arterial infusion therapy as preoperative treatment before hepatectomy were excluded from this study. According to the definition of a multidisciplinary international consensus (21), liver metastases found at the time of the diagnosis were defined as synchronous, and those found after treatment of the primary lesion were defined as metachronous.

The patients were classified into three groups as follows: i) Patients who did not receive preoperative chemotherapy; ii) patients who received short-term preoperative chemotherapy for $<6$ months; and iii) patients who received long-term preoperative chemotherapy for $\geq 6$ months.

The present study was performed in compliance with the principles expressed in the Declaration of Helsinki and was approved by the Ethics committee of Osaka City University (approval no. 3853). Written informed consent was obtained from all participants prior to the study.

Methods. Peripheral blood samples were obtained from patients within two weeks before surgery ans were used to calculate the absolute peripheral lymphocyte count. The differential white blood cell count was analyzed using an XE-5000 hematology analyzer (Sysmex Corporation) according to the manufacturer's protocol. CD3, a pan-T lymphocyte marker, and CD8, a cytotoxic T lymphocyte marker, which both serve key role in antitumor immunity, were used as targets for the evaluation of TIL infiltration. Immunohistochemistry staining was performed using surgically resected specimens of liver metastases as previously described (14). The outline of the evaluation method is as follows. All specimens treated with $10 \%$ formalin fixation for $24-48 \mathrm{~h}$ at room temperature and paraffin embedding were cut into $4-\mu \mathrm{m}$-thick sections to perform immunohistochemistry staining. Sections were deparaffined and rehydrated, and subjected to endogenous peroxidase blocking in $1 \% \mathrm{H}_{2} \mathrm{O}_{2}$ solution in methanol for $15 \mathrm{~min}$ at room temperature. Antigen retrieval was carried out by autoclaving the sections at $105^{\circ} \mathrm{C}$ for $10 \mathrm{~min}$ in Dako Target Retrieval Solution (Dako; Agilent Technologies, Inc.). Serum blocking was performed with antibody in $10 \%$ normal rabbit serum (Histofine SAB-PO (M) kit; cat. no. 424022; Nichirei Biosciences) for $10 \mathrm{~min}$ at room temperature. After $\mathrm{H}_{2} \mathrm{O}_{2}$ and serum blocking, the slides were incubated with the primary monoclonal mouse anti-human antibodies anti-CD3 (1:400; clone F7.2.38; Dako; Agilent Technologies, Inc.) and anti-CD8 (1:200; clone C8/144B; Dako; Agilent Technologies, Inc.) at $4^{\circ} \mathrm{C}$ overnight. The secondary antibody was biotin-labeled rabbit anti-mouse $\operatorname{IgG}+\operatorname{IgA}+\operatorname{IgM}$ antibody (1:500; Histofine SAB-PO (M) kit; cat. no. 424022; Nichirei Biosciences). Signal detection was performed with a DAB kit (Histofine simple stain kit; Nichirei Biosciences). The sections were counterstained with hematoxylin. TILs in the inner part of the tumor margin of liver metastases were observed with an optical microscope, and the number of TILs was counted in a randomly selected field at a magnification of $x 400$. The mean values obtained in five different areas were used for the data analysis. Examples of CD8 ${ }^{+}$TILs and $\mathrm{CD}^{+}{ }^{+}$TILs are presented in Fig. $1 \mathrm{~A}$ and B.

The specimens were pathologically classified according to the Union for International Cancer Control Tumor-Node-Metastasis classification of malignant tumors (8thedition) (https://www.uicc. org/resources/tnm/publications-resources). All patients were followed up until death or November 30, 2018. The relapse-free survival (RFS) was determined from the date of hepatectomy to the date of relapse, the date of death from any cause, the date of loss to follow-up, or the 30th of November 2018, whichever occurred first. The overall survival (OS) was determined from the date of hepatectomy to the date of death, the date of loss to follow-up, or the 30th of November 2018, whichever occurred first. 
A

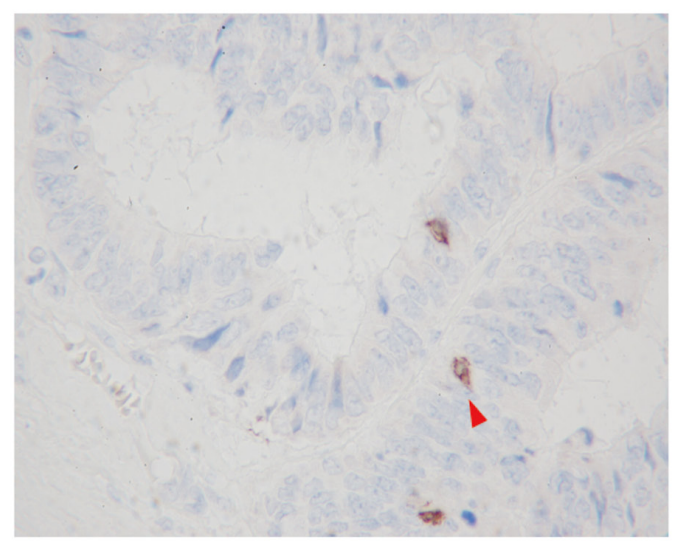

B

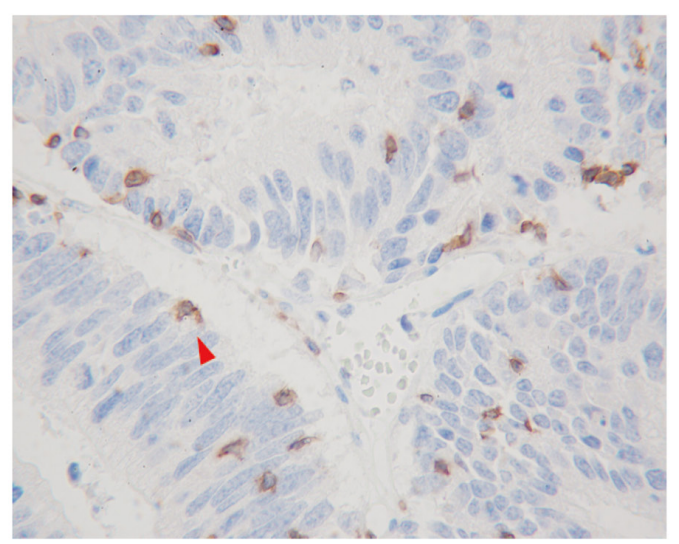

Figure 1. Immunohistochemical detection of (A) CD8-positive TILs (red arrow in A) and (B) CD3-positive TILs (red arrow in B) by x400 high-power field. TILs, tumor-infiltrating lymphocytes.

Statistical analyses. Analyses were performed using the JMP13 software (version 13.2.0; SAS Institute, Inc.) and the SPSS 25.0 statistical software program (IBM Corp.). Differences between the density of TILs and absolute peripheral lymphocyte count were analyzed using Pearson's correlation coefficient, and differences in the density of TILs between the recurrence group and the recurrence-free group after hepatectomy were analyzed using Wilcoxon's rank sum test. A receiver operating characteristic (ROC) curve was used to determine the appropriate cut-off value for the density of TILs. All patients were then classified into two groups according to the numbers of CD8 ${ }^{+}$TILs and $\mathrm{CD}^{+}{ }^{+}$TILs. The significance of the correlations between the density of TILs and the clinicopathological characteristics was analyzed using Yates' Chi-square test and Fisher's exact test (only these two tests were analyzed using the SPSS software program). The duration of the survival was estimated using the Kaplan-Meier method and differences in the survival curves were analyzed using the log-rank test. $\mathrm{P}<0.05$ was considered to indicate a statistically significant difference.

\section{Results}

Relationship between $C D 8^{+} / C D 3^{+} T I L$ infiltration in colorectal cancer liver metastases and the absolute peripheral lymphocyte count. In patients with resected colorectal liver metastases, there was a positive correlation between the density of CD8 ${ }^{+}$TILs and the density of CD3 ${ }^{+}$TILs in liver metastases
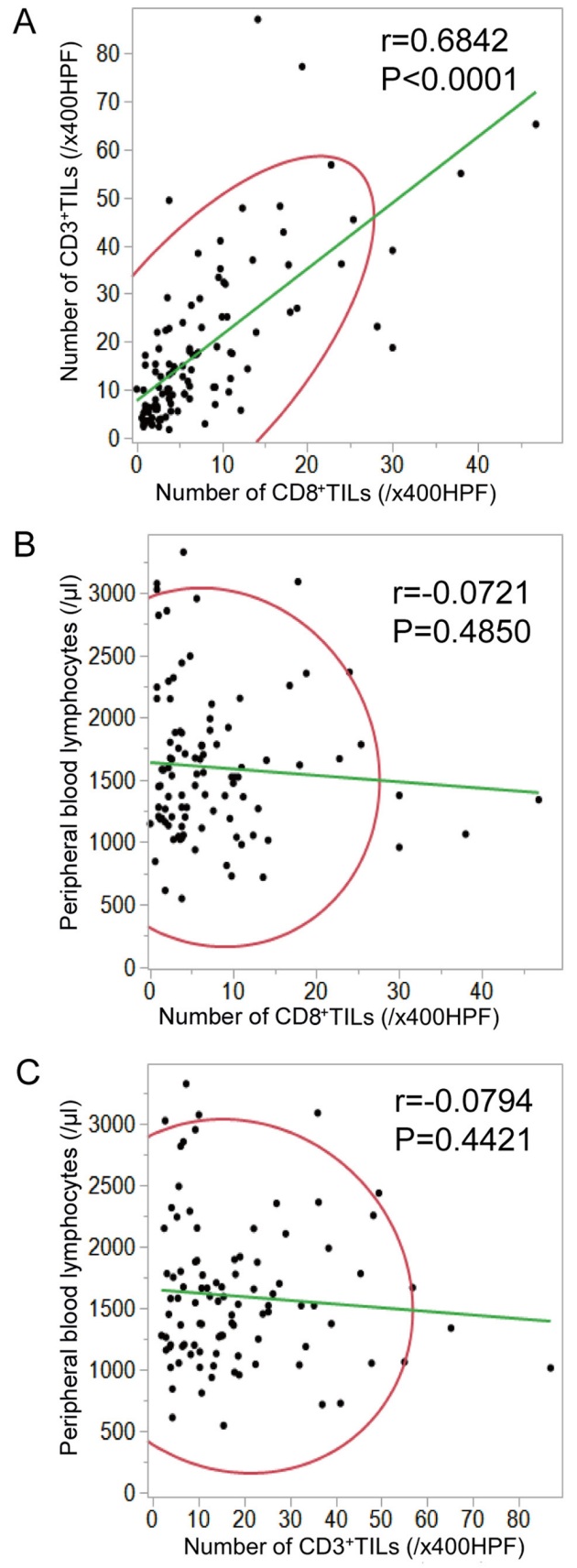

Figure 2. Relationship between $\mathrm{CD}^{+} / \mathrm{CD}^{+} \mathrm{TIL}$ infiltration in colorectal cancer liver metastases and the absolute peripheral lymphocyte count. (A) In patients with resected colorectal liver metastases, there was a positive correlation between the density of $\mathrm{CD}^{+}$TILs and that of $\mathrm{CD} 3^{+}$TILs in liver metastases $(r=0.6842, \mathrm{P}<0.0001)$. (B and $\mathrm{C}$ ) The density of TILs in liver metastases did not correlate with the absolute peripheral lymphocyte count (CD8, r=-0.0721, P=0.4850; CD3, r=-0.0794, P=0.4421). TILs, tumor-infiltrating lymphocytes.

$(\mathrm{r}=0.6842 ; \mathrm{P}<0.0001 ;$ Fig. $2 \mathrm{~A})$. The density of $\mathrm{CD}^{+} / \mathrm{CD}^{+} \mathrm{TILs}$ in liver metastases was not correlated with the absolute peripheral lymphocyte count (CD8, $\mathrm{r}=-0.0721, \mathrm{P}=0.4850$; CD3, r=-0.0794, P=0.4421; Fig. 2B and C, respectively).

Comparison of TIL infiltration in colorectal cancer liver metastases between the recurrence group and the recurrence-free group after hepatectomy. In patients with resected colorectal liver metastases, the density of CD8 ${ }^{+}$TILs 

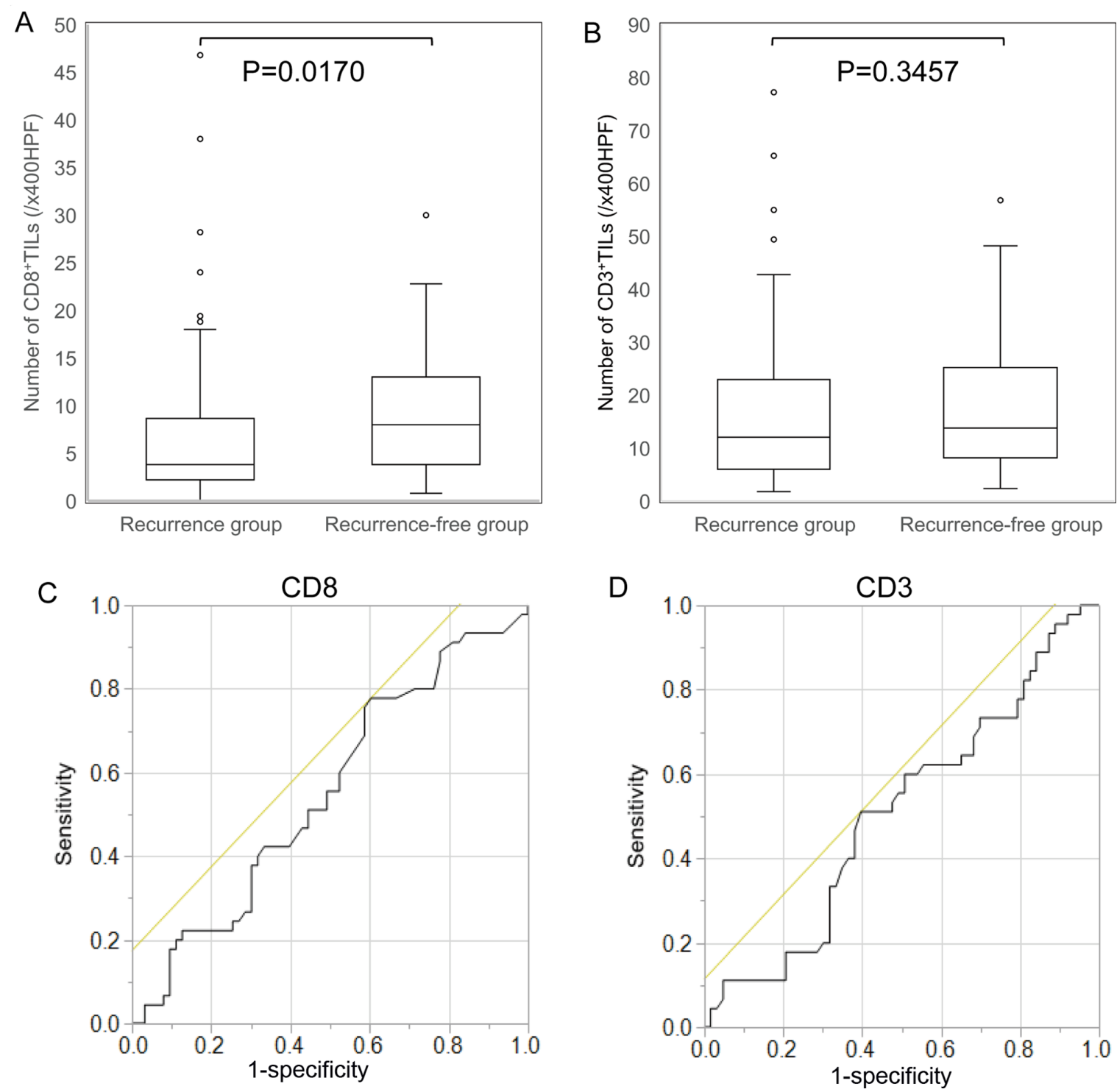

Figure 3. Comparison of TIL infiltration in colorectal cancer liver metastases between the recurrence group and the recurrence-free group after hepatectomy, and ROC curve for determination of the cut-off value of the density of TILs for predicting the prognosis. (A) In patients with resected colorectal liver metastases, the density of $\mathrm{CD}^{+}$TILs in liver metastases in the recurrence-free group was significantly higher than that in the recurrence group $(\mathrm{P}=0.017)$. (B) There were no significant differences between the density of CD3 ${ }^{+}$TILs in the recurrence-free group and the recurrence group (P=0.3457). $(\mathrm{C}$ and $\mathrm{D}) \mathrm{ROC}$ curve analysis of the density of TILs. The five-year overall survival was treated as the state variable, and the density of TILs was treated as the test variable. The ROC curve showed that $3.4 / \mathrm{x} 400 \mathrm{HPF}$ was the most appropriate cut-off value for the density of CD8+TIL (area under the curve, 0.5460 ; sensitivity, 0.7778 ; specificity, 0.3906) and 10.6/x400HPF was the most appropriate cut-off value for the density of CD3 ${ }^{+}$TIL (area under the curve, 0.5090 ; sensitivity, 0.5111 ; specificity, 0.6032). TILs, tumor-infiltrating lymphocytes; ROS, receiver operating characteristic; HPF, high-power field.

in liver metastases in the recurrence-free group was significantly higher than that in the recurrence group [median values: recurrence-free group 8.0 vs. recurrence group 3.8; /x400 high-power field (HPF); Fig. 3A]. Conversely, there was no significant difference between the density of CD3+TILs in the recurrence-free group and the recurrence group (Fig. 3B).

Cut-off value of the density of TILs for predicting the prognosis of patients with colorectal cancer liver metastasis. We considered the five-year OS as the state variable and the density of $\mathrm{CD}^{+} / \mathrm{CD}^{+}$TILs as the test variable. The ROC curve showed that $3.4 / \mathrm{x} 400 \mathrm{HPF}$ was the most appropriate cut-off value for the density of CD8 ${ }^{+}$TIL (area under the curve, 0.5460; sensitivity, 0.7778; specificity, 0.3906; Fig. 3C) and that $10.6 / \mathrm{x} 400 \mathrm{HPF}$ was the most appropriate cut-off value for the density of CD3 ${ }^{+}$TIL (area under the curve, 0.5090; sensitivity, 0.5111; specificity, 0.6032; Fig. 3D). Subsequently, we classified the patients into a high-TIL group and low-TIL group.

Relationship between TIL infiltration in colorectal cancer liver metastases and clinicopathological factors or the prognosis after hepatectomy.

Analyses of patients with colorectal liver metastases. The high-CD8 ${ }^{+}$TIL group was significantly associated with $\mathrm{CEA} \leq 5 \mathrm{ng} / \mathrm{ml}(\mathrm{P}=0.025)$ but not with other clinicopathological characteristics (Table I). The high-CD8 ${ }^{+}$TIL group exhibited a significantly better RFS than the low-CD8+TIL group [high-CD8 ${ }^{+}$TIL median survival time (MST), 24.8 months; low-CD8+TIL MST, 11.5 months; P=0.0045; Fig. 4A]. Similarly, the high-CD8 ${ }^{+}$TIL group exhibited a better OS than 


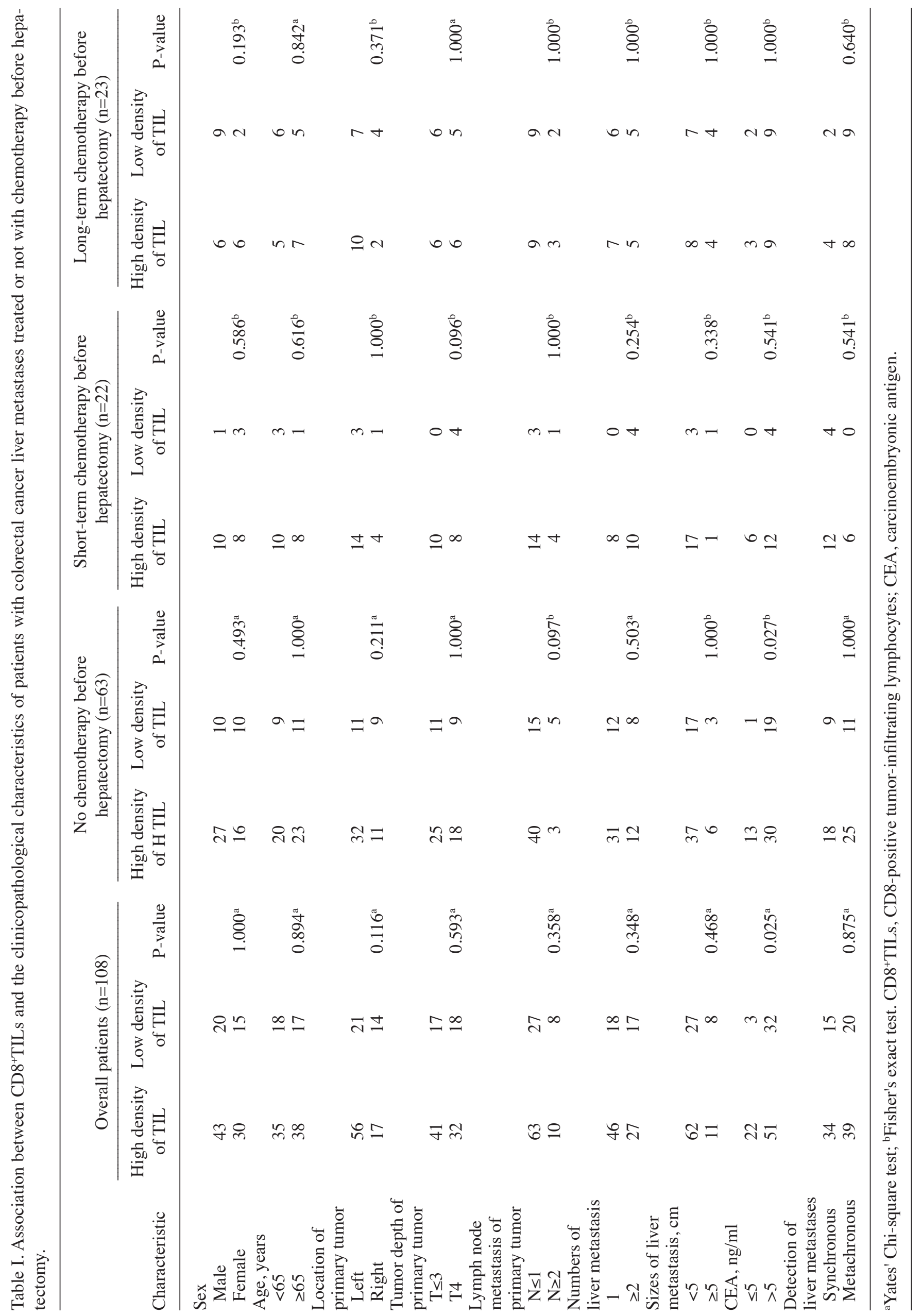



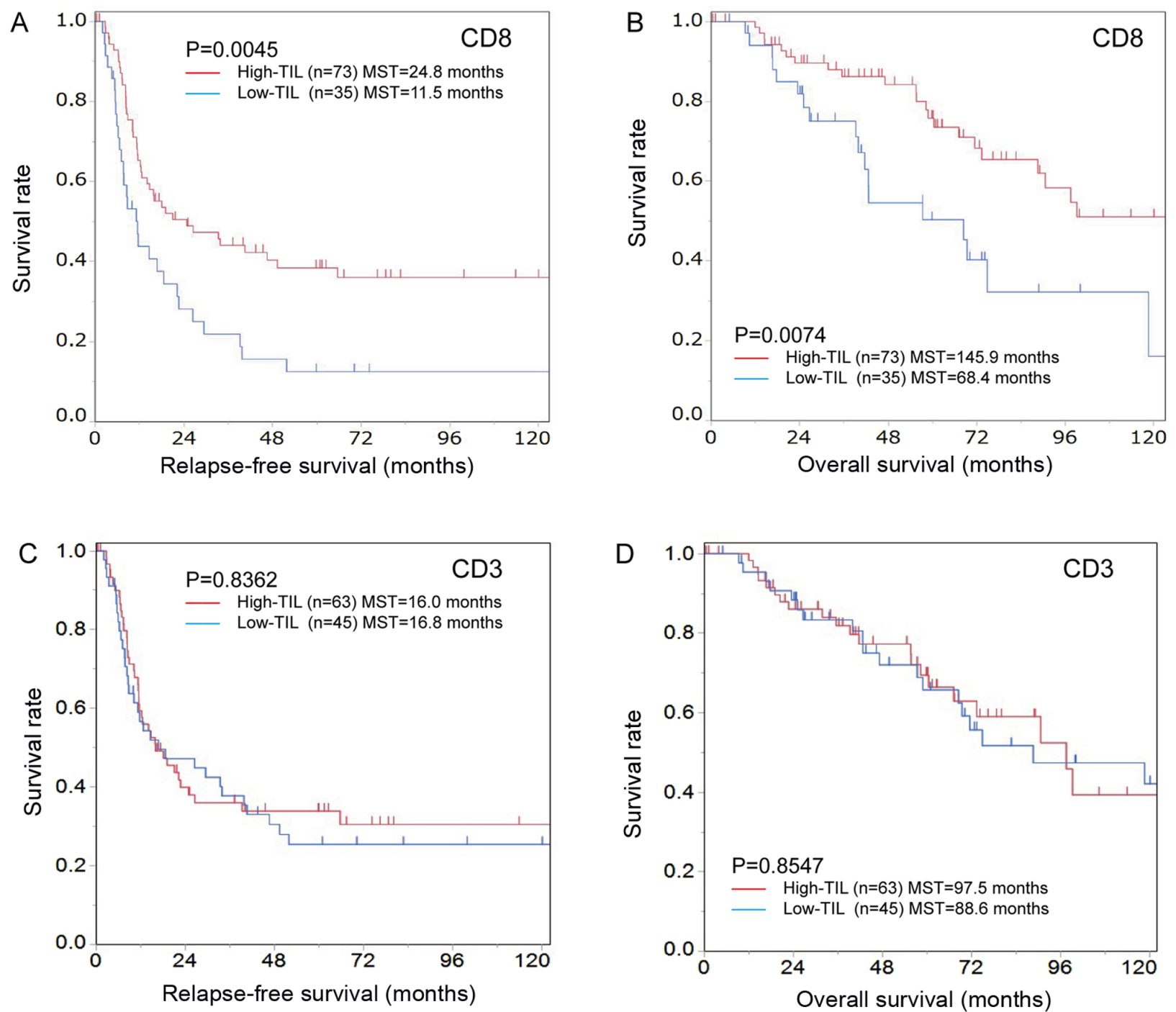

Figure 4. Kaplan-Meier survival curves for the relapse-free survival and overall survival according to the number of CD8-positive TILs in patients with colorectal cancer liver metastases. (A) Relapse-free and (B) overall survival were significantly better in the high-CD8 ${ }^{+}$TIL group compared with the low-CD ${ }^{+}$TIL group (relapse-free survival, $\mathrm{P}=0.0045$; overall survival, $\mathrm{P}=0.0074)$. (C) There were no significant differences between the high- and low-CD3 ${ }^{+} \mathrm{TIL}$ groups in relapse-free $(\mathrm{P}=0.8362)$ or $(\mathrm{D})$ overall survival $(\mathrm{P}=0.8547)$. TILs, tumor-infiltrating lymphocytes.

the low-CD8+TIL group (high-CD8+TILs MST, 145.9 months; low-CD8+TILs MST, 68.4 months; P=0.0074; Fig. 4B).

The high-CD3 ${ }^{+}$TIL group was significantly associated with left-sided colorectal cancer $(\mathrm{P}=0.048)$ and CEA $\leq 5 \mathrm{ng} / \mathrm{ml}(\mathrm{P}=0.006)$ but not with other clinicopathological characteristics (Table II). There were no significant differences between the high- and low-CD $3^{+} \mathrm{TIL}$ groups regarding the RFS or OS (Fig. 4C and D).

Subgroup analyses by preoperative chemotherapy. i) Subgroup analyses in the group without preoperative chemotherapy. Among patients who did not receive preoperative chemotherapy, the high-CD $8^{+} \mathrm{TIL}$ group was significantly associated with $\mathrm{CEA} \leq 5 \mathrm{ng} / \mathrm{ml}(\mathrm{P}=0.027)$ but not with other clinicopathological characteristics (Table I). The degree of CD8 ${ }^{+}$TIL infiltration was significantly associated with the RFS (high-CD8 ${ }^{+}$TIL MST, 40.6 months; low-CD8 ${ }^{+}$TIL MST, 11.5 months; $\mathrm{P}=0.0031$; Fig. 5A). Furthermore, a high $\mathrm{CD} 8^{+}$TIL presence tended to have a better effect on the OS than a low CD8 ${ }^{+}$TIL presence (high-CD8 ${ }^{+}$TIL MST, not available; low-CD8+TIL MST, 68.4 months; $\mathrm{P}=0.0537$; Fig. 5B).
The high-CD3 ${ }^{+} \mathrm{TIL}$ group showed a significant association with $C E A \leq 5 \mathrm{ng} / \mathrm{ml}(\mathrm{P}=0.040)$ but not with other clinicopathological characteristics (Table II). The RFS and OS of the high- and low-CD3 ${ }^{+} \mathrm{TIL}$ groups did not differ to a statistically significant extent (Fig. 6A and B).

ii) Subgroup analyses in the short-term chemotherapy group. The degree of CD $8^{+}$TIL infiltration was not correlated with the clinicopathological characteristics in the short-term chemotherapy group (Table I). The degree of CD8 ${ }^{+}$TIL infiltration was significantly associated with the RFS (high-CD8+TIL MST, 14.7 months; low-CD8+TIL MST, 5.4 months; $\mathrm{P}=0.0003$ ) and $\mathrm{OS}$ (high-CD8 ${ }^{+}$TIL MST, 90.6 months; low-CD8+TIL MST, 17.7 months; $\mathrm{P}=0.0005$; Fig. 5C and D).

The high-CD3 ${ }^{+}$TIL group showed a significant association with the tumor depth of the primary tumor $(\mathrm{T} \leq 3 ; \mathrm{P}=0.040)$ but not with other clinicopathological characteristics (Table II). The degree of $\mathrm{CD}^{+}$TIL infiltration was significantly associated with RFS (high-CD3 ${ }^{+}$TIL MST, 16.4 months; low-CD3 ${ }^{+}$TIL MST, 5.4 months; $\mathrm{P}=0.0017$ ) and 


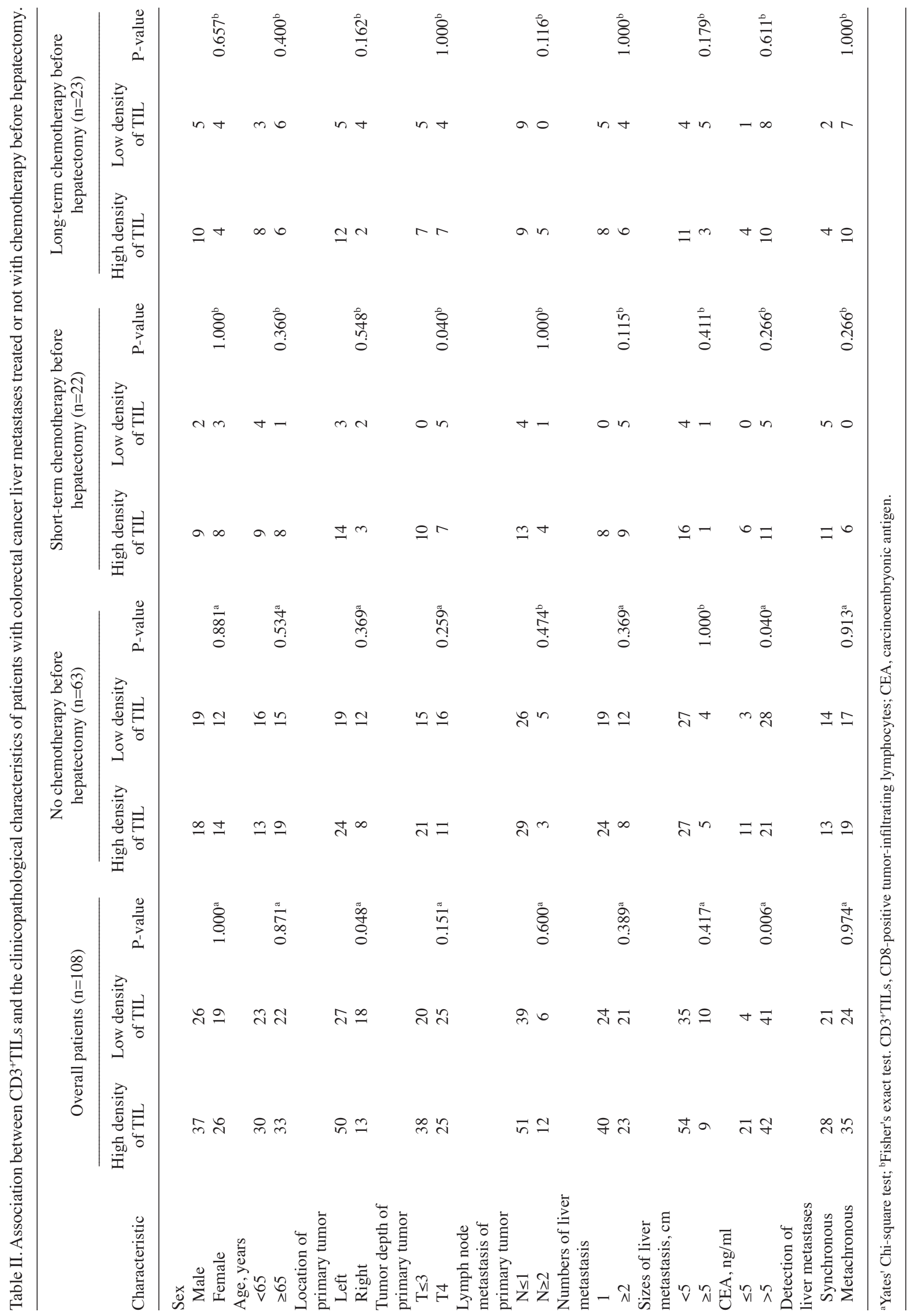


A

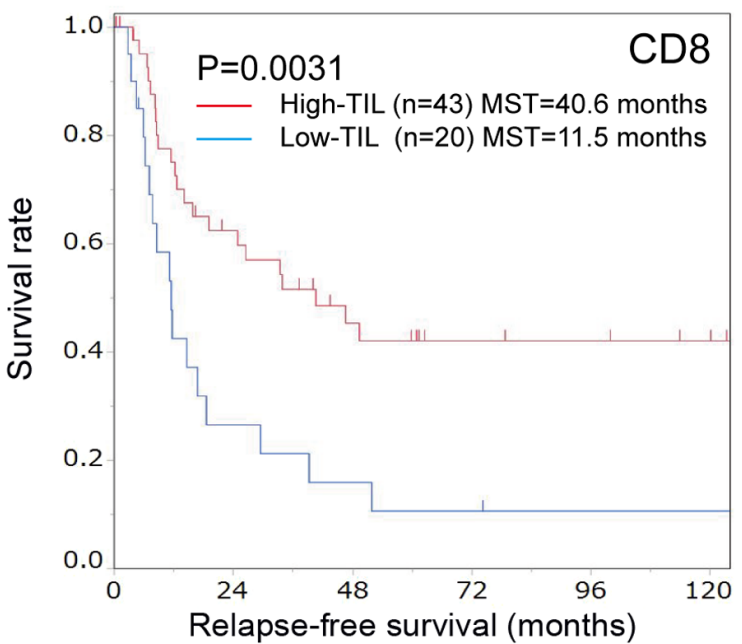

C

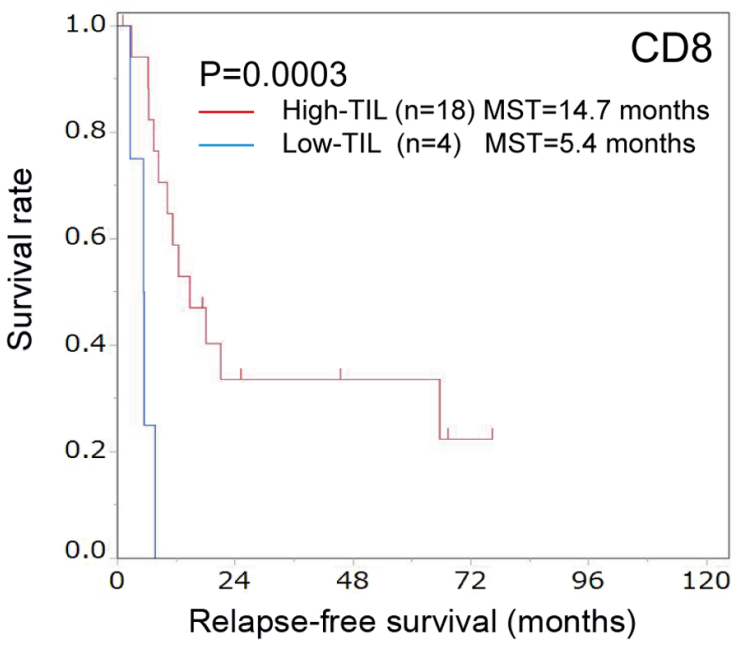

E

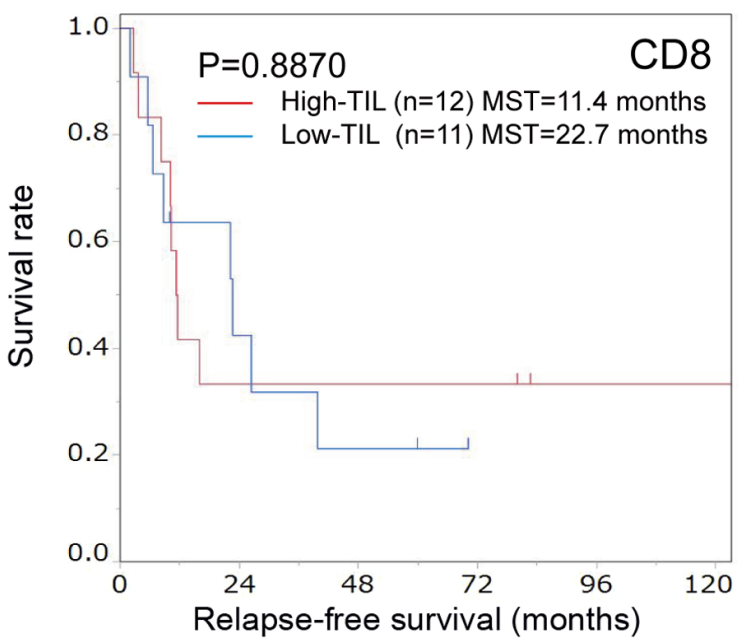

B

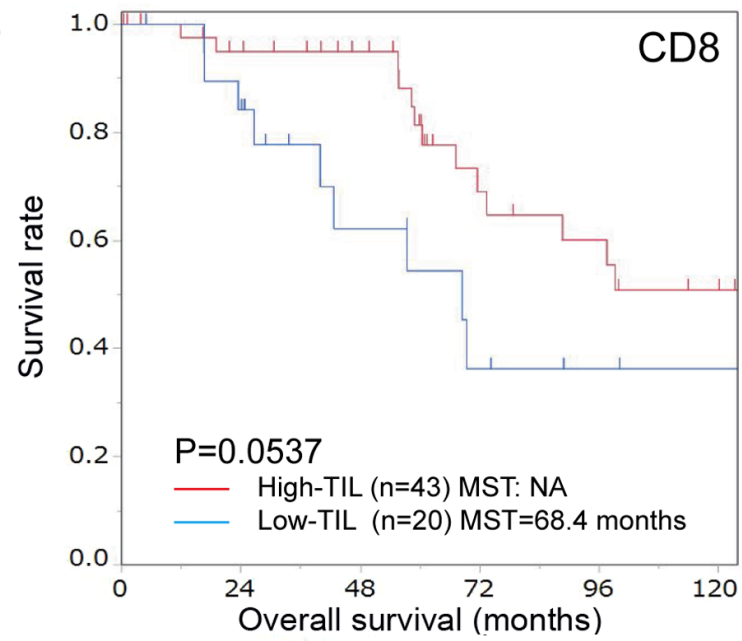

D

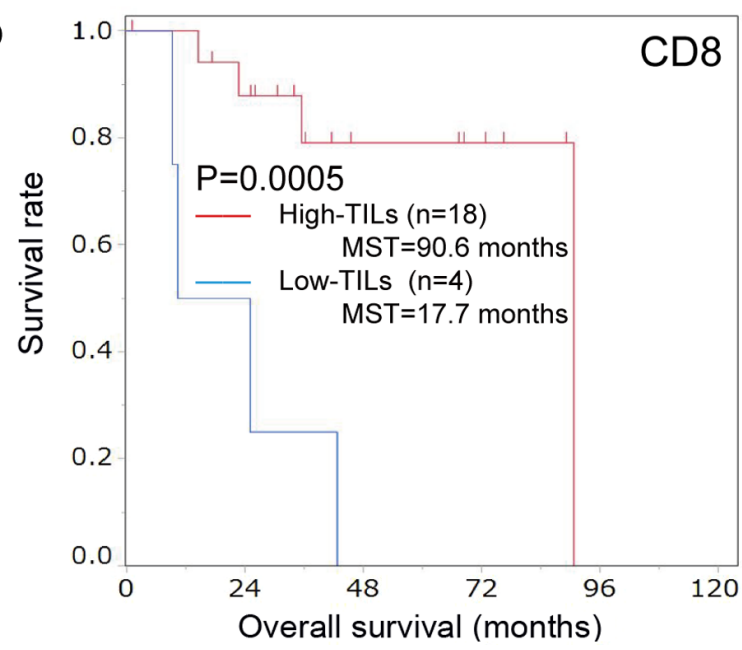

$\mathrm{F}$

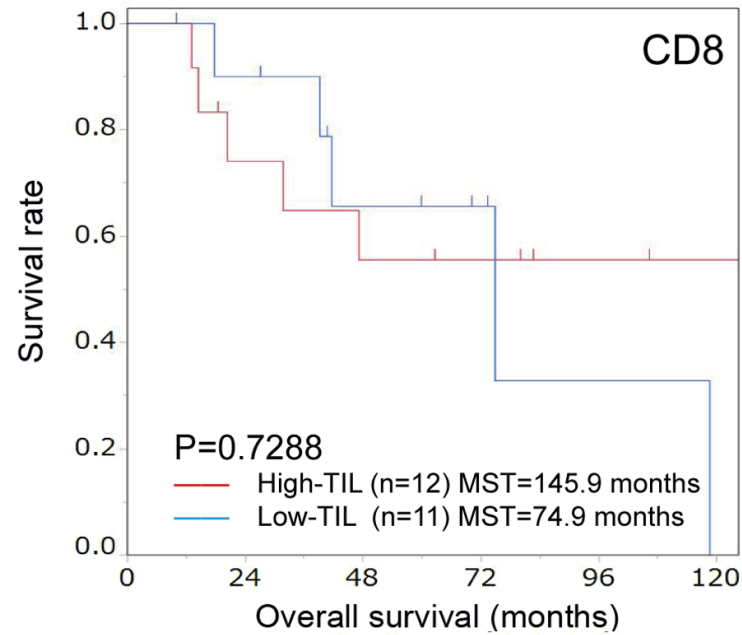

Figure 5. Kaplan-Meier survival curves for the relapse-free survival and overall survival according to the number of CD8-positive TILs in patients with colorectal cancer liver metastasis with/without chemotherapy before hepatectomy. Kaplan-Meier survival curves for the (A) relapse-free survival and (B) overall survival in the group that did not receive chemotherapy before hepatectomy. Relapse-free survival was significantly better in the high-CD8 ${ }^{+}$TIL group compared with the low-CD8 ${ }^{+}$TIL group $(\mathrm{P}=0.0031)$. High CD8-positive TIL infiltration tended to have a better effect on overall survival than low infiltration $(\mathrm{P}=0.0537)$. Kaplan-Meier survival curves for the $(\mathrm{C})$ relapse-free survival and (D) overall survival in the group treated with short-term chemotherapy before hepatectomy. The relapse-free and overall survival were significantly better in the high-CD8 ${ }^{+}$TIL group than in the low-CD8 ${ }^{+}$TIL group (relapse-free survival, $\mathrm{P}=0.0003$; overall survival, $\mathrm{P}=0.0005)$. Kaplan-Meier survival curves for the $(\mathrm{E})$ relapse-free survival and ( $\mathrm{F}$ ) overall survival in the group treated with long-term chemotherapy before hepatectomy. There were no significant differences between the high- and low-CD8 ${ }^{+}$TIL groups (relapse-free survival, $\mathrm{P}=0.8870$; overall survival, $\mathrm{P}=0.7288$ ). TILs, tumor-infiltrating lymphocytes.

OS (high-CD3+TIL MST, 90.6 months; low-CD3+TIL MST, 25.0 months; $\mathrm{P}=0.0132$; Fig. $6 \mathrm{C}$ and $\mathrm{D})$. iii) Subgroup analyses in the long-term chemotherapy group. The degree of $\mathrm{CD}^{+}{ }^{+} \mathrm{TIL}$ infiltration was not correlated with 
A

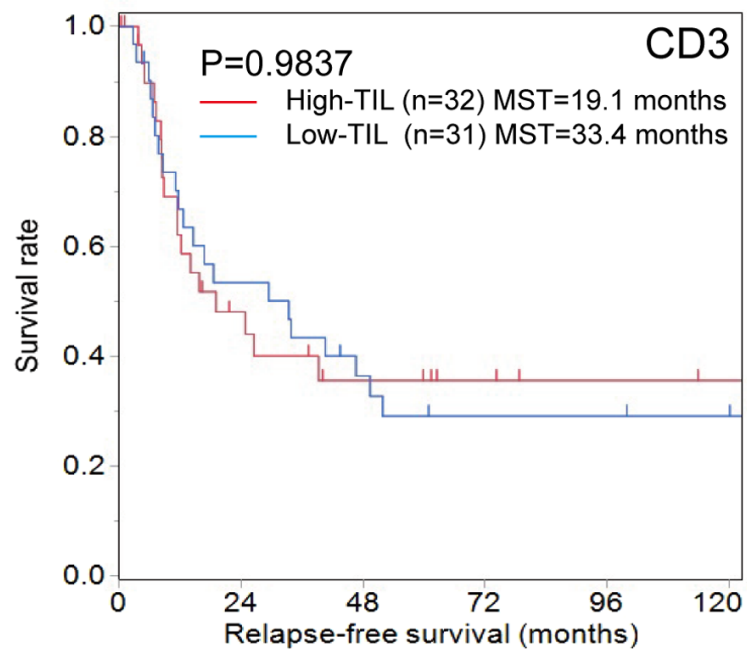

C

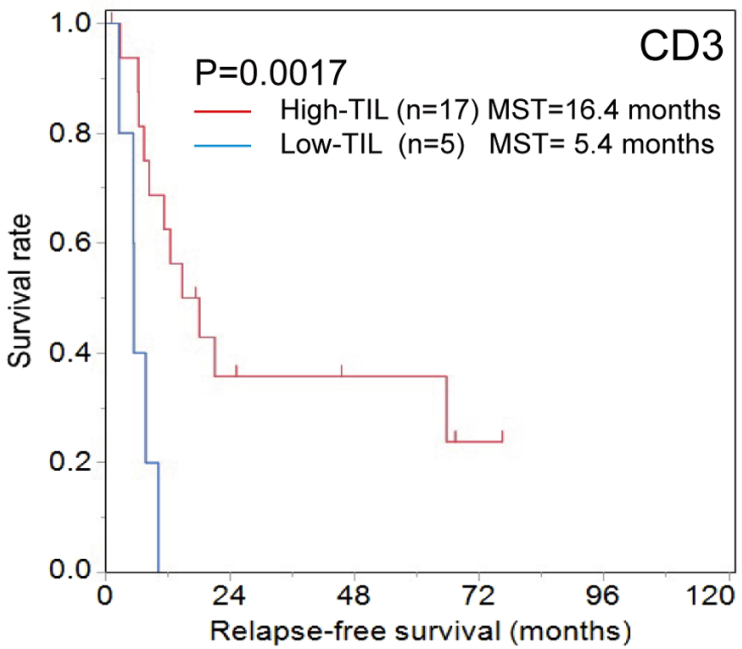

E

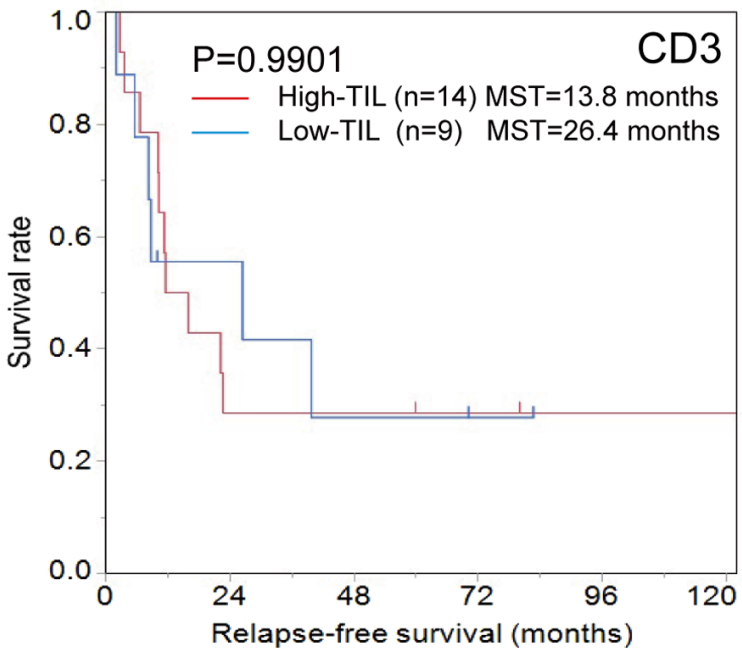

B

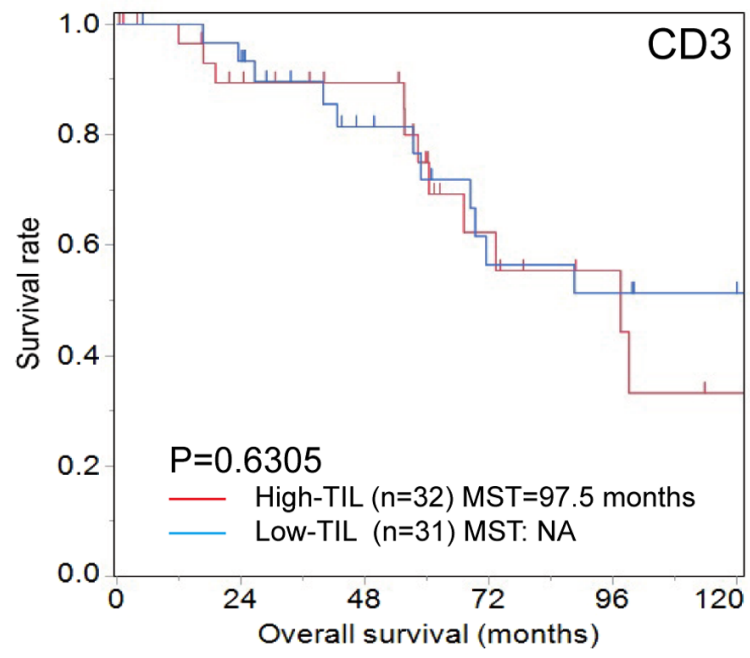

D

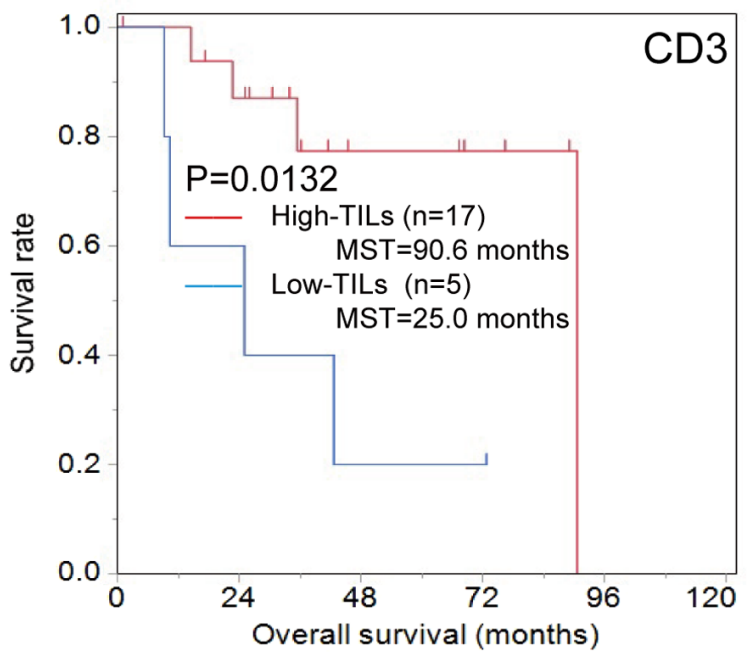

F

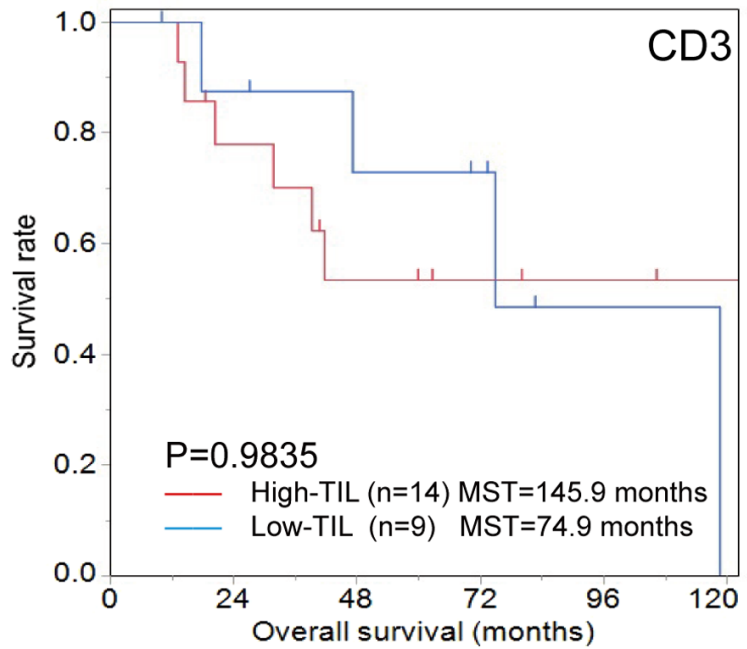

Figure 6. Kaplan-Meier survival curves for relapse-free survival and overall survival according to the number of CD3-positive TILs in patients with colorectal cancer liver metastasis with/without chemotherapy before hepatectomy. Kaplan-Meier survival curves for (A) relapse-free survival and (B) overall survival in the group that did not receive chemotherapy before hepatectomy. There were no significant differences between the high- and low-CD3 ${ }^{+}$TIL groups (relapse-free survival, $\mathrm{P}=0.9837$; overall survival, $\mathrm{P}=0.6305$ ). Kaplan-Meier survival curves for $(\mathrm{C})$ relapse-free survival and (D) overall survival in the group treated with short-term chemotherapy before hepatectomy. The relapse-free and overall survival in the high-CD3 ${ }^{+} \mathrm{TIL}$ group were significantly better than in the low-CD3 ${ }^{+}$TIL group (relapse-free survival, $\mathrm{P}=0.0017$; overall survival, $\mathrm{P}=0.0132$ ). Kaplan-Meier survival curves for (E) relapse-free survival and ( $\mathrm{F}$ ) overall survival in the group treated with long-term chemotherapy before hepatectomy. There were no significant differences between the high- and low-CD ${ }^{+}$ TIL groups (relapse-free survival, $\mathrm{P}=0.9901$; overall survival, $\mathrm{P}=0.9835$ ). TILs, tumor-infiltrating lymphocytes

clinicopathological characteristics in the long-term chemotherapy group (Table I). There were no significant differences between the high- and low-CD8+TIL groups regarding the RFS or OS (Fig. 5E and F). 
The degree of $\mathrm{CD}^{+}{ }^{+}$TIL infiltration was not associated with clinicopathological characteristics (Table II). The RFS and OS of the high- and low-CD3 ${ }^{+}$TIL groups did not differ to a statistically significant extent (Fig. 6E and F).

\section{Discussion}

The present study demonstrated that the degree of CD8 ${ }^{+}$TIL infiltration in colorectal cancer liver metastases was significantly lower in the recurrence group compared with the recurrence-free group following hepatectomy, indicating that the density of CD8 ${ }^{+}$TILs may strongly influence the therapeutic effect on and prognosis of colorectal liver metastasis. Furthermore, the local immune status in colorectal cancer liver metastases was correlated with the prognosis after curative hepatectomy in the group without preoperative chemotherapy as well as the group with short-term preoperative chemotherapy. These findings were in accordance with the results from previous studies regarding the primary tumor of colorectal cancer (10-12). Conversely, the degree of TILs was not correlated with patient prognosis in the long-term preoperative chemotherapy group.

Previous studies have reported that the prognosis of various types of carcinoma, including colorectal cancer, is strongly influenced by the cancer microenvironment, including TILs (22-24). The degree of TIL infiltration in the primary tumor of colorectal cancer is positively correlated with recurrence and survival after curative resection $(22,25,26)$ as well as with the effects of radiotherapy and chemotherapy $(13,14,27)$. While relatively few studies have focused on local immunity in distant metastases of colorectal cancer compared to that in the primary tumor of colorectal cancers, a high degree of TIL infiltration in liver metastases reportedly leads to good OS (28). The present study also demonstrated that a large number of CD8 ${ }^{+}$TILs in colorectal cancer liver metastasis was significantly correlated with a good RFS and OS in patients without preoperative chemotherapy or with short-term preoperative chemotherapy.

Most TILs are T cells (10), which mediate adaptive immune responses by targeting antigens expressed by tumor cells (29). However, there are various subsets of $\mathrm{T}$ cells with different functions. Th1 cells have been reported to activate $\mathrm{CD} 8+\mathrm{T}$ cells and enhance the anti-tumor immune response, while Th 2 cells seem to suppress anti-tumor immunity by activating B cells or producing IL-10 (14,23). The functions of Th17 and Treg cells in antitumor immunity have been controversial, but they reportedly activate anti-tumor immunity or promote tumor growth $(14,23)$. $\mathrm{CD}^{+} \mathrm{T}$ cells have been reported to kill tumor cells directly via granzymes/perforin and the tumor necrosis factor superfamily and to play a key role in anti-tumor immunity, influencing therefore patient prognosis $(25,30,31)$.

In the present study, in patients who did not undergo preoperative chemotherapy before hepatectomy or in those who underwent short-term preoperative chemotherapy, the high-CD8 ${ }^{+}$TIL group in colorectal cancer liver metastasis was associated with a prolonged OS and RFS. Conversely, the degree of TIL infiltration of colorectal cancer liver metastases was not associated with the prognosis in patients with a long-term preoperative chemotherapy. According to previous studies, long-term chemotherapy may change the local immunity in liver metastases and reduce the prognostic value of the density of TILs (20,32-38). There may be some possible causes to this observation. Firstly, the dysfunction of lymphocytes may be involved. Long-term chemotherapy induces a reduction in the absolute number of lymphocytes and prevents the induction of lymphocytes to secondary lymphoid organs, resulting in decreased lymphocyte activation and thereby weakening the $\mathrm{T}$ cell immune response (32). A study by Wu et al (20) using an experimental mouse model demonstrated that the short-term administration of fluorouracil can increase the proliferation and cytotoxicity of $\mathrm{CD}^{+} \mathrm{T}$ cells, while repeated administration causes a decrease in the proliferation and cytotoxicity of $\mathrm{CD}^{+} \mathrm{T}$ cells. Furthermore, the absolute number of immune cells may recover relatively quickly after the discontinuation of chemotherapy; however, the antitumor immune function of immune cells remains diminished for a prolonged period with repeated chemotherapy $(20,33)$. Therefore, it was speculated that the number of $\mathrm{T}$ cells in the local tumor microenvironment may not always be associated with the prognosis in cases treated with long-term chemotherapy, as the function of T cells decreases after repeated chemotherapy. Secondly, immune escape mechanisms may be involved. Repeated chemotherapy may increase the expression of immune checkpoint molecules, such as programmed death-ligand 1 (PD-L1) in tumor cells, dendritic cells or macrophages, resulting in immune exhaustion and consequently impaired lymphocyte-mediated immunity $(34,35)$. PD-L1 is known to bind to PD-1 expressed on T cells and suppress the function of TILs. However, while chemotherapy activates local immunity, including TILs, an immunosuppressive mechanism called adaptive immune resistance occurs when PD-L1 expression is upregulated in response to interferon $-\gamma$ secreted by $\mathrm{CD}^{+} \mathrm{T}$ cells and suppresses the function of TILs (36-38). In long-term chemotherapy, the activation of this mechanism results in the overexpression of PD-L1 and counteracts the immunological effects of TILs $(37,38)$, thus the degree of TIL infiltration may not necessarily correlate with therapeutic effects.

The present study reported no correlation between the degree of TIL infiltration in liver metastases and the absolute peripheral lymphocyte count. Regarding the reason for this absence of correlation, the absolute peripheral lymphocyte count was not evaluated for subgroups with different functions, including T cells, B cells and NK cells. Furthermore, various factors, such as suppressive immune cells, structural abnormalities in the wall of newly formed microvessels, chemokines and cytokines and intratumoral fibrosis, which may affect the recruitment of peripheral lymphocytes into tumors (39-41), were not under consideration in the present study. Therefore, the local immune status of the tumor may not necessarily be associated with the absolute peripheral lymphocyte count. Similarly, we demonstrated that the prognostic ability of CD3 was lower than that of CD8. CD3, a pan-T lymphocyte marker, includes various subsets, such as Th1, Th2, Th17, regulatory $\mathrm{T}$ cell and cytotoxic $\mathrm{T}$ lymphocyte. These subsets have different functions, resulting in the poor prognostic ability of CD3 ${ }^{+}$TILs.

This study presented several limitations. Firstly, this study was a retrospective, single-center study with a relatively small number of patients. Secondly, we examined only CD8 ${ }^{+}$TILs 
as immune factors that may affect patient prognosis; however, other immunocompetent cells, including other TILs subtypes, tumor-associated macrophages, dendritic cells and myeloid-derived suppressor cells, antigen presentation capability, immune activation markers and immune checkpoint markers and chemokines involved in the local recruitment of immunocompetent cells were not considered in the present study. Thirdly, chemotherapy for at least six months was defined as long-term chemotherapy in this study; however, how long chemotherapy should be continued for to reduce lymphocyte immunity is unclear.

In summary, the present study demonstrated that the degree of $\mathrm{CD}^{+} \mathrm{TIL}$ infiltration in colorectal cancer liver metastases may be correlated with that in the primary lesion as well as with patient prognosis. However, in patients who received long-term chemotherapy prior to surgery, the degree of TIL infiltration was not necessarily associated with the prognosis because the function of TILs may be decreased.

\section{Acknowledgements}

Not applicable.

\section{Funding}

No funding was received.

\section{Availability of data and materials}

The datasets used and/or analyzed during the current study are available from the corresponding author on reasonable request

\section{Authors' contributions}

EW and MS conceived and designed the experiments. EW performed the experiments and was a major contributor in analyzing the data. EW and MS contributed to reagents, materials and analytical tools. EW and MS wrote the paper. HN, TF, YI, YO, SK, HT and KM were contributors in revising the design of the experiments and in analyzing the data. MS, HN, TF, YI, YO, SK, HT and KM reviewed and validated the manuscript. EW and MS confirm the authenticity of all the raw data. All authors read and approved the final manuscript.

\section{Ethics approval and consent to participate}

This study was performed in compliance with the principles expressed in the Declaration of Helsinki and was approved by the Ethics Committee of Osaka City University (approval no. 3853). Written informed consent was obtained from all participants in this study.

\section{Patient consent for publication}

Not applicable.

\section{Competing interests}

The authors declare that they have no competing interests.

\section{References}

1. World Health Organization (WHO): WHO report on cancer: setting priorities, investing wisely and providing care for all. World Health Organization, Geneva, 2020.

2. Watanabe T, Muro K, Ajioka Y, Hashiguchi Y, Ito Y, Saito Y, Hamaguchi $T$, Ishida $H$, Ishiguro $M$, Ishihara $S$, et al: Japanese Society for Cancer of the Colon and Rectum (JSCCR) guidelines 2016 for the treatment of colorectal cancer. Int J Clin Oncol 23: 1-34, 2018.

3. Niederhuber JE: Colon and rectum cancer. Patterns of spread and implications for workup. Cancer 71 (Suppl 12): 4187-4192, 1993.

4. Wakabayashi T, Hibi T, Yoneda G, Iwao Y, Sawada Y, Hoshino H, Uemura S, Ban D, Kudo A, Takemura Y, et al: Predictive model for survival after liver resection for noncolorectal liver metastases in the modern era: A Japanese multicenter analysis. J Hepatobiliary Pancreat Sci 26: 441-448, 2019.

5. Zarour LR, Anand S, Billingsley KG, Bisson WH, Cercek A, Clarke MF, Coussens LM, Gast CE, Geltzeiler CB, Hansen L, et al: Colorectal Cancer Liver Metastasis: Evolving Paradigms and Future Directions. Cell Mol Gastroenterol Hepatol 3: 163-173, 2017.

6. Abdalla EK, Vauthey JN, Ellis LM, Ellis V, Pollock R, Broglio KR, Hess K and Curley SA: Recurrence and outcomes following hepatic resection, radiofrequency ablation, and combined resection/ablation for colorectal liver metastases. Ann Surg 239: 818-825, discussion 825-827, 2004.

7. Kato T, Yasui K, Hirai T, Kanemitsu Y, Mori T, Sugihara K, Mochizuki $\mathrm{H}$ and Yamamoto J: Therapeutic results for hepatic metastasis of colorectal cancer with special reference to effectiveness of hepatectomy: Analysis of prognostic factors for 763 cases recorded at 18 institutions. Dis Colon Rectum 46 (Suppl 10): S22-S31, 2003

8. Gooden MJ, de Bock GH, Leffers N, Daemen T and Nijman HW: The prognostic influence of tumour-infiltrating lymphocytes in cancer: A systematic review with meta-analysis. Br J Cancer 105: 93-103, 2011.

9. Zhang D, He W, Wu C, Tan Y, He Y, Xu B, Chen L, Li Q and Jiang J: Scoring System for Tumor-Infiltrating Lymphocytes and Its Prognostic Value for Gastric Cancer. Front Immunol 10: 71-71, 2019.

10. Ropponen KM, Eskelinen MJ, Lipponen PK, Alhava E and Kosma V-M: Prognostic value of tumour-infiltrating lymphocytes (TILs) in colorectal cancer. J Pathol 182: 318-324, 1997.

11. Canna K, McArdle PA, McMillan DC, McNicol AM, Smith GW, McKee RF and McArdle CS: The relationship between tumour T-lymphocyte infiltration, the systemic inflammatory response and survival in patients undergoing curative resection for colorectal cancer. Br J Cancer 92: 651-654, 2005.

12. Mei Z, Liu Y, Liu C, Cui A, Liang Z, Wang G, Peng H, Cui L and Li C: Tumour-infiltrating inflammation and prognosis in colorectal cancer: Systematic review and meta-analysis. Br J Cancer 110: 1595-1605, 2014.

13. Shibutani M, Maeda K, Nagahara H, Fukuoka T, Iseki Y, Matsutani S, Kashiwagi S, Tanaka H, Hirakawa K and Ohira M: Tumor-infiltrating Lymphocytes Predict the Chemotherapeutic Outcomes in Patients with Stage IV Colorectal Cancer. In Vivo 32: 151-158, 2018.

14. Matsutani S, Shibutani M, Maeda K, Nagahara H, Fukuoka T, Nakao S, Hirakawa K and Ohira M: Significance of tumor-infiltrating lymphocytes before and after neoadjuvant therapy for rectal cancer. Cancer Sci 109: 966-979, 2018.

15. Teng F, Mu D, Meng X, Kong L, Zhu H, Liu S, Zhang J and Yu J: Tumor infiltrating lymphocytes (TILs) before and after neoadjuvant chemoradiotherapy and its clinical utility for rectal cancer. Am J Cancer Res 5: 2064-2074, 2015.

16. Anitei MG, Zeitoun G, Mlecnik B, Marliot F, Haicheur N, Todosi AM, Kirilovsky A, Lagorce C, Bindea G, Ferariu D, et al: Prognostic and predictive values of the immunoscore in patients with rectal cancer. Clin Cancer Res 20: 1891-1899, 2014.

17. Lee WS, Kang M, Baek JH, Lee JI and Ha SY: Clinical impact of tumor-infiltrating lymphocytes for survival in curatively resected stage IV colon cancer with isolated liver or lung metastasis. Ann Surg Oncol 20: 697-702, 2013.

18. Shibutani M, Maeda K, Nagahara H, Fukuoka T, Matsutani S, Kashiwagi S, Tanaka H, Hirakawa K and Ohira M: A comparison of the local immune status between the primary and metastatic tumor in colorectal cancer: A retrospective study. BMC Cancer 18: 371, 2018. 
19. Kito A, Tanaka K, Fujimaki H, Nakazawa M, Togo S, Minami $M$ and Shimada H: Tumor doubling time and local immune response to hepatic metastases from colorectal cancer. J Surg Oncol 96: 525-533, 2007.

20. Wu Y, Deng Z, Wang H, Ma W, Zhou C and Zhang S: Repeated cycles of 5-fluorouracil chemotherapy impaired anti-tumor functions of cytotoxic T cells in a CT26 tumor-bearing mouse model. BMC Immunol 17: 29, 2016.

21. Adam R, de Gramont A, Figueras J, Kokudo N, Kunstlinger F, Loyer E, Poston G, Rougier P, Rubbia-Brandt L, Sobrero A, et al: Managing synchronous liver metastases from colorectal cancer: A multidisciplinary international consensus. Cancer Treat Rev 41: 729-741, 2015.

22. Galon J, Costes A, Sanchez-Cabo F, Kirilovsky A, Mlecnik B, Lagorce-Pagès C, Tosolini M, Camus M, Berger A, Wind $\mathrm{P}$, et al: Type, density, and location of immune cells within human colorectal tumors predict clinical outcome. Science 313: 1960-1964, 2006.

23. Fridman WH, Pagès F, Sautès-Fridman C and Galon J: The immune contexture in human tumours: Impact on clinical outcome. Nat Rev Cancer 12: 298-306, 2012.

24. Galon J, Angell HK, Bedognetti D and Marincola FM: The continuum of cancer immunosurveillance: Prognostic, predictive, and mechanistic signatures. Immunity 39: 11-26, 2013.

25. Pagès F, Kirilovsky A, Mlecnik B, Asslaber M, Tosolini M, Bindea G, Lagorce C, Wind P, Marliot F, Bruneval P, et al: In situ cytotoxic and memory $\mathrm{T}$ cells predict outcome in patients with early-stage colorectal cancer. J Clin Oncol 27: 5944-5951, 2009.

26. Eriksen AC, Sørensen FB, Lindebjerg J, Hager H, dePont Christensen R, Kjær-Frifeldt S and Hansen TF: The Prognostic Value of Tumor-Infiltrating lymphocytes in Stage II Colon Cancer. A Nationwide Population-Based Study. Transl Oncol 11: 979-987, 2018.

27. Halama N, Michel S, Kloor M, Zoernig I, Pommerencke T, von Knebel Doeberitz M, Schirmacher P, Weitz J, Grabe N and Jäger D: The localization and density of immune cells in primary tumors of human metastatic colorectal cancer shows an association with response to chemotherapy. Cancer Immun 9: 1, 2009.

28. Katz SC, Pillarisetty V, Bamboat ZM, Shia J, Hedvat C, Gonen M, Jarnagin W, Fong Y, Blumgart L, D'Angelica M, et al: $\mathrm{T}$ cell infiltrate predicts long-term survival following resection of colorectal cancer liver metastases. Ann Surg Oncol 16 2524-2530, 2009.

29. Boon T, Cerottini JC, Van den Eynde B, van der Bruggen $P$ and Van Pel A: Tumor antigens recognized by T lymphocytes. Annu Rev Immunol 12: 337-365, 1994.

30. Cullen SP, Brunet M and Martin SJ: Granzymes in cancer and immunity. Cell Death Differ 17: 616-623, 2010.
31. Medema JP, de Jong J, Peltenburg LT, Verdegaal EM, Gorter A, Bres SA, Franken KL, Hahne M, Albar JP, Melief CJ, et al: Blockade of the granzyme B/perforin pathway through overexpression of the serine protease inhibitor PI-9/SPI-6 constitutes a mechanism for immune escape by tumors. Proc Natl Acad Sci USA 98: 11515-11520, 2001.

32. Liu L, Zhao L, Yang Y, Gao J, Hu C, Guo B and Zhu B: Cytotoxic chemotherapy reduces $\mathrm{T}$ cell trafficking to the spleen by downregulating the expression of $\mathrm{C}-\mathrm{C}$ motif chemokine ligand 21 and $\mathrm{C}-\mathrm{C}$ motif chemokine ligand 19. Oncol Lett 16: 5013-5019, 2018

33. Mackall CL: T-cell immunodeficiency following cytotoxic antineoplastic therapy: A review. Stem Cells 18: 10-18, 2000.

34. Zhang P, Ma Y, Lv C, Huang M, Li M, Dong B, Liu X, An G, Zhang $\mathrm{W}$, Zhang J, et al: Upregulation of programmed cell death ligand 1 promotes resistance response in non-small-cell lung cancer patients treated with neo-adjuvant chemotherapy. Cancer Sci 107: 1563-1571,2016.

35. Guo L, Song P, Xue X, Guo C, Han L, Fang Q, Ying J, Gao S and $\mathrm{Li}$ W: Variation of Programmed Death Ligand 1 Expression After Platinum-based Neoadjuvant Chemotherapy in Lung Cancer. J Immunother 42: 215-220, 2019.

36. Khairallah AS, Genestie C, Auguste A and Leary A: Impact of neoadjuvant chemotherapy on the immune microenvironment in advanced epithelial ovarian cancer: Prognostic and therapeutic implications. Int J Cancer 143: 8-15, 2018.

37. Xiao B, Peng J, Wang Y, Deng Y, Ou Q, Wu X, Lin J, Pan Z and Zhang L: Prognostic value of tumor infiltrating lymphocytes combined with PD-L1 expression for patients with solitary colorectal cancer liver metastasis. Ann Transl Med 8: 1221, 2020.

38. Thompson ED, Zahurak M, Murphy A, Cornish T, Cuka N, Abdelfatah E, Yang S, Duncan M, Ahuja N, Taube JM, et al: Patterns of PD-L1 expression and CD8 T cell infiltration in gastric adenocarcinomas and associated immune stroma. Gut 66: 794-801, 2017.

39. Joyce JA and Fearon DT: T cell exclusion, immune privilege, and the tumor microenvironment. Science 348: 74-80, 2015.

40. Salmon H, Franciszkiewicz K, Damotte D, Dieu-Nosjean MC, Validire P, Trautmann A, Mami-Chouaib F and Donnadieu E: Matrix architecture defines the preferential localization and migration of T cells into the stroma of human lung tumors. J Clin Invest 122: 899-910, 2012.

41. Slaney CY, Kershaw MH and Darcy PK: Trafficking of T cells into tumors. Cancer Res 74: 7168-7174, 2014.

(c) $($ ) This work is licensed under a Creative Commons cc) Attribution-NonCommercial-NoDerivatives 4.0 International (CC BY-NC-ND 4.0) License. 